\title{
Post Surgical Review of Bariatric Surgery Patients: A Feasibility Study of Multidisciplinary Follow Up Using Videoconferencing
}

\author{
Emma Morrow*, Duff Morrison Bruce, Elizabeth Bruce, Cathy Dorrian and Fiona Sim \\ Clinical Psychologist, Obesity Services, Rosehill Annexe, Foresterhill Site, Cornhill Road, Aberdeen AB25 $2 Z G$, UK
}

\begin{abstract}
:
Background: Bariatric surgery is an effective means of managing weight and reducing medical co-morbidities in the obese patient. However, psychological difficulties are common and adequate multidisciplinary support is vital for postsurgical success. Videoconferencing is potentially a vehicle for the delivery of support to patients residing in remote areas.

Methods: Ten patients were invited to attend a videolink session utilising videoconferencing technology to allow clinicians to connect audio-visually with patients from two remote locations. Seven patients attended. A Consultant Surgeon, Clinical Psychologist and Specialist Dietitian reviewed the patients. Patients and clinicians completed a post-session evaluation questionnaire and commented on their experience.

Results: The videolink session was evaluated as acceptable and useful to both patients and clinicians. Patients and clinicians were satisfied with the user-friendliness of the technology.

Conclusion: Videolink technology may be a viable and accessible means of delivering specialist multidisciplinary input to bariatric patients. Further research is necessary.
\end{abstract}

Keywords: Bariatric surgery, obesity, videoconferencing.

\section{INTRODUCTION}

The evidence supporting bariatric surgery as the most effective means of managing severe obesity (BMI $>40 \mathrm{~kg} / \mathrm{m}^{2}$ ) is becoming increasingly compelling. It has been demonstrated that surgical intervention allows obese individuals to lose weight and reduces or eliminates obesity-related comorbidities such as hypertension, diabetes, hyperlipidemia and obstructive sleep apnoea [1]. In addition to the weight loss and health benefits, bariatric surgery has been shown to have a positive impact on quality of life [2], depression [3] and anxiety [4].

Pre-surgery psychiatric difficulties are common in the treatment-seeking bariatric population, most frequently adjustment disorders, anxiety disorders, mood disorders and eating disorders. Research has shown that the lifetime prevalence of Axis I disorders in surgery seeking patients is up to $66 \%$ [5]. It has been demonstrated, however, that psychopathology generally improves following bariatric surgery. However, improvements may dissipate over time and patients may experience reoccurrence of previous difficulties [6]. Therefore, close monitoring of post surgical patients is recommended [7]. The obesity surgery patient may face massive challenges in response to the level of weight loss achieved; learning to cope with vastly reduced food consumption, coping with a dramatic change in appearance, relationship changes, weight plateaus and dealing with ex-

\footnotetext{
*Address correspondence to this author at the Obesity Services, Rosehill Annexe, Foresterhill Site, Cornhill Road, Aberdeen AB25 2ZG, UK; Tel: 00441224559365; E-mail: emma.morrow@nhs.net, mail@emmamorrow.co.uk
}

cess skin [8]. The post surgical assessment of patients ideally should take account of not only medical and surgical issues, but also dietetic difficulties, psychiatric status, psychosocial functioning, eating patterns and quality of life. Thus, multidisciplinary support is of crucial importance in managing this potentially complex clinical picture.

Positive surgical outcome has been found to be related to the provision of ongoing therapeutic contact [9]. Pre-surgical psychological assessment has become routine in this specialist area, but post-surgical psychological support is less common. Psychological support may help patients cope with the aftermath of surgery. In some cases, patients may feel a sense of failure if they do not feel able to make the surgery a success without assistance and this may limit their tendency to ask for help from professionals. It is therefore important that multi-disciplinary support is readily accessible to patients.

Many factors may determine how well supported patients are; resource, staffing, motivation and also geographical location. Due to the highly specialist nature of bariatric surgery, it is likely that bariatric teams are physically located in hospital settings, usually in urban areas. This means that patients living in rural areas are required to travel to attend appointments.

Videoconferencing technology has been successfully utilised in a range of clinical settings. Psychiatric and psychological assessments have been conducted via videolink [1012] and, although the research in this area is sparse, results indicate that videolink is a viable alternative to face-to-face contact [13]. Ongoing therapeutic interventions using video- 
link have also been investigated with promising outcomes in terms of acceptability to both patients and clinicians [14]. Telemedicine in general has been demonstrated to be a feasible alternative to face to face contact across a wide range of clinical settings [15]. In a review of videoconferencing within the realms of psychiatry and psychology, it has been concluded that videoconferencing is at least as effective as face to face contacts [16], although methodological issues continue to limit the generalisability of many studies in this area.

In the complex and highly specialist arena of bariatric surgery, videoconferencing technology may allow the expertise of health professionals to reach remote and rural locations. This type of outreach is particularly vital for this patient-group, who frequently experience mobility problems which limit their own capacity to travel and attend appointments outside their local area.

There are no studies to date investigating the feasibility of using videoconferencing technology with bariatric surgery patients. However, it has been suggested that telecommunications technology may be used successfully to generate behaviour change in obese patients [17] and support weight management programmes [18-22]. Additionally, it has it has been shown that the use of telemedicine can in fact be a more effective means of improving obesity outcomes than routine hospital based interventions [23]. The current feasibility study involved the development of a remote multidisciplinary follow up clinic for patients living far from the specialist bariatric centre. The aim was to provide an accessible and acceptable service that would allow patients to make contact with team members in order to discuss any post-surgical issues - be it surgical, dietetic or psychological.

\section{MATERIALS AND METHODOLOGY}

\section{Patients}

Ten post-procedure bariatric surgery patients were contacted by telephone and invited to attend a follow up review session. The protocol for the session was explained to patients explicitly. Two patients were unable to attend due to other commitments and one patient became ill prior to her appointment and cancelled. Seven patients were reviewed.

\section{Clinicians}

Patients were seen by a Consultant Surgeon (face-toface), a Senior Dietitian (via videolink) and a Clinical Psychologist (via videolink). All clinicians had previous experience of using videoconferencing technology in clinical settings. The session was conducted jointly, with all clinicians present. It was agreed that this was the most time-efficient method of reviewing the patients.

\section{Technological Specifications}

The connection was made using digital telephone lines (ISDN) with a band width of $384 \mathrm{kbit} / \mathrm{s}$. The videoconferencing equipment in Aberdeen was a Tandberg 95Edge system. In Inverness, the equipment was a Tandberg 990MXP system.

\section{Procedure}

Patients were asked to attend their local acute general hospital (Inverness). On arrival for their appointment, pa- tients were met in person by the project facilitator and the bariatric co-ordinator. Patients were again reminded of the protocol for the appointment; the surgeon would be meeting them in person, with the psychologist and dietitian linking in to the appointment from the specialist centre for bariatric surgery (Aberdeen) via videolink. On entry to the clinic room, the surgeon introduced each clinician and explained the format of the appointment; each clinician would ask questions in turn to avoid more than one person speaking at one time. It was explained to the patient that there would be a slight time delay when speaking over the videolink. The patient was encouraged to inform the clinicians if they were unable to hear any questions asked. The patient was also reassured that the dietitian and psychologist were the only people at the far end site and that the session was not being recorded. The follow up session lasted between 30-45 minutes. Each clinician asked the patient questions related to their progress following surgery. Any difficulties in each of the clinical areas (dietetic, psychological, surgical) were identified and recommendations were made. Finally, patients were invited to ask any questions. Following the session, patients were given an evaluation questionnaire by the project facilitator. They were also verbally asked how they had generally found the experience. Each clinician completed a questionnaire pertaining to each clinical session. The clinicians discussed each patient following the session and agreed on the pertinent details to be communicated to the patient's GP.

\section{Measures}

The evaluation questionnaires included a patient questionnaire and a clinician scale. The patient scale was a standard 19 item likert-type scale (1=strongly disagree $5=$ strongly agree) which included questions from three domains: quality of the consultation, user friendliness of the system and overall patient satisfaction. Clinicians completed a 5-item questionnaire about their experience of the session. Patient demographics, type of surgery and outcomes were also recorded.

\section{RESULTS}

Ten patients were contacted. Two declined to attend due to having other commitments. Eight patients agreed to attend but one cancelled at late notice due to illness. Seven patients were reviewed via multidisciplinary videolink consultation. Six patients were female and one was male. The median age of patients was 54 years and the median number of days since surgery was 500 . Four patients had undergone insertion of a gastric band only. One patient had undergone gastric band insertion with a later revision to gastric bypass. One patient had undergone insertion of an intragastric balloon and subsequently had a gastric bypass. One patient had an intragastric balloon inserted and subsequently underwent a sleeve gastrectomy operation.

Median, minimum and maximum values for the patient questionnaire are displayed in Table $\mathbf{1}$ and for the clinician questionnaire in Table 2.

Overall patients and clinicians found the videolink consultation to be acceptable and useful. There were no issues with the user-friendliness of the technology. Patient comments are displayed below. 
Table 1. Patient Questionnaire

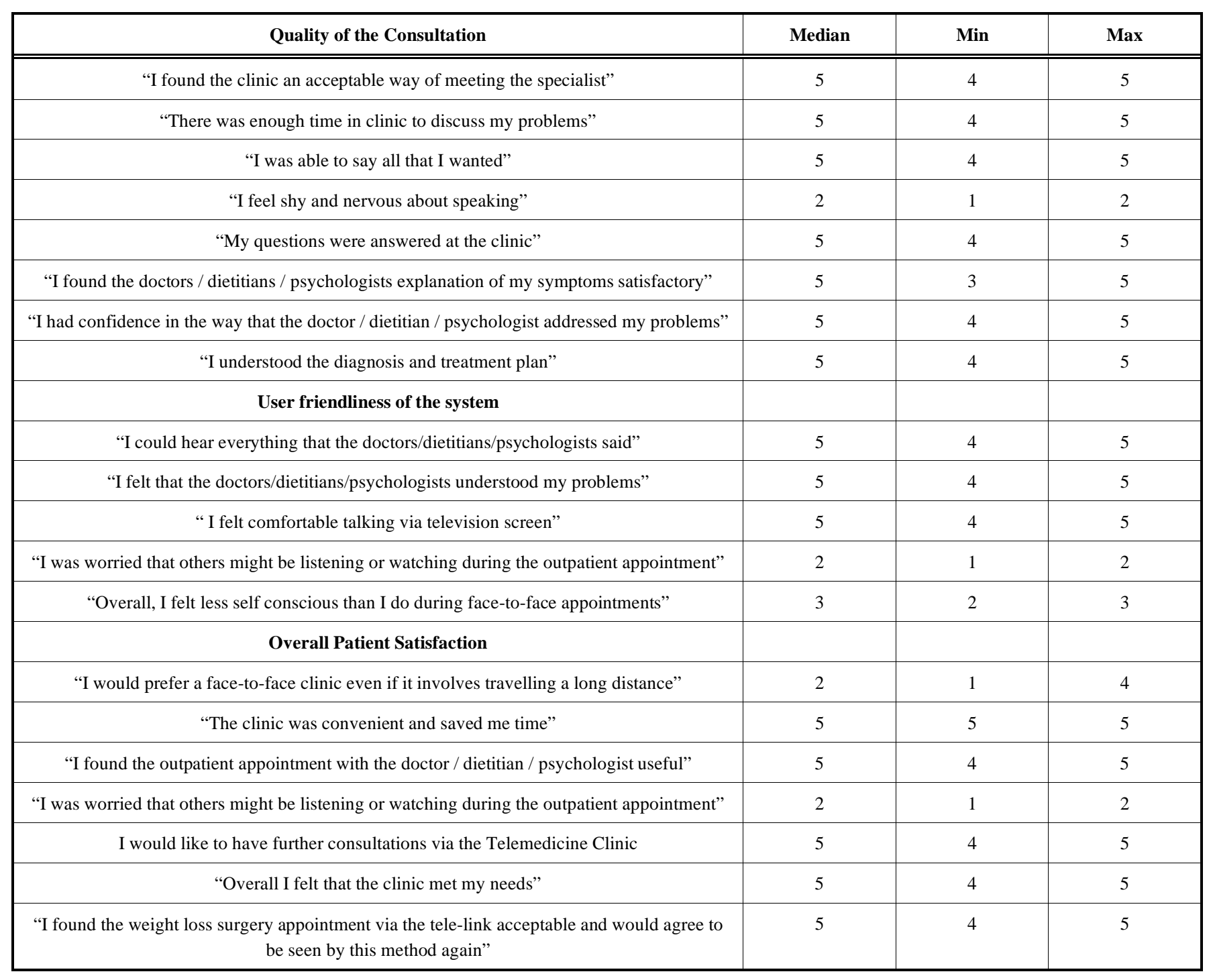

Table 2. Clinician Questionnaire

\begin{tabular}{|c|c|c|}
\hline & Median & Min \\
\hline \hline "The clinic was an acceptable method of reviewing the patient" & 5 & 5 \\
\hline "The clinic was as effective as a face-to-face interview" & 5 & 5 \\
\hline "There was sufficient time to assess the patient and arrive at a management plan" & 5 & 5 \\
\hline "The clinic visit had a useful outcome" & 5 & 5 \\
\hline "Rapport was as easily established with the patient as in a face-to-face interview" & 5 & 5 \\
\hline
\end{tabular}

\section{Patient Comments}

I found the videolink to be a method of linking in with the health team without the need to travel long distances for short appointments. It has allowed me to express my views, ask questions and be given advice and guidance. I was able to engage with all members present in a meaningful way. On a slightly different note, weight loss surgery has been of huge benefit to me. It has been life changing and impacted on all areas of my being. From a health perspective I am no longer diabetic or have high blood pressure. Both conditions impacted on my general health and well being. I have increased confidence in my day to day life and am more able to live my life to the full. I could not have achieved this level of weight loss and improved health and well being without surgery.

Very useful to see all staff at the same time. Noticeable time delay when speaking was slightly irritating. I was apprehensive about the meeting but enjoyed the experience. 
Now more confident re weight loss. The convenience of not having the 9 hour round trip to Aberdeen was excellent. Hope this becomes more frequent. Thank you.

I find this way of an appointment good. It saves me a $B$ \& $B$ and train fare. I would like face to face at some meetings because it is a slightly different way of relating to the dietitian etc. I did feel a little of a distance between myself and Aberdeen which one doesn't have in a face to face. On the whole I think the plan is a good idea. (patient called later to say that although she really enjoyed today's session, she really enjoys face to face with the surgeon as she has built up a good relationship with him).

The following observations were made by the project facilitator:

one patient wished to use the technology to help set up a patient support group.

one patient wished to use the videolink technology in her own working life.

one patient commented on how helpful it was having a local appointment as she did not need to pay for travel costs to Aberdeen or arrange childcare for her young child.

One patient stated that she found having the three clinicians at the same appointment very beneficial. She stated that she had failed to attend some previous appointments in Aberdeen due to the journey involved.

One patient stated that she would usually need to stay overnight in Aberdeen when attending appointments so the local appointment saved her money.

\section{DISCUSSION}

This feasibility study indicates that videoconferencing technology can be considered as a possible method of postbariatric surgery follow up. The method was acceptable to patients and clinicians, irrespective of clinical background. Videoconferencing provides an accessible and convenient means of accessing complex patients in remote and rural areas. The results of the feasibility study suggest that patients from other remote areas may be able to access specialist bariatric surgery teams using videolink. This may serve to increase equity of access to follow up post-surgical services and in turn may enhance the patient experience in order to provide optimal outcomes, in terms of weight loss and quality of life. Travelling to remote areas may be a waste of valuable clinician resource and therefore videolink can minimise travel for clinicians, as well as patients. Videolink might assist in providing a more financially viable service for patients and may also have cost-savings for health providers. Due to the small sample size of the current study, findings should be treated with some caution and clearly further research is required to verify the feasibility of using videoconferencing technology as an alternative to face-toface contact with bariatric surgery patients.

During clinical discussions where decisions are being made, the decision reached does not always meet with patient's expectations. This is particularly the case with surgical patients where hopes and expectations of surgical results can be unrealistic. It was observed that one patient involved in the study did not appear satisfied with the outcome of the session. It would be interesting to consider whether satisfaction with the outcome of a consultation influences the patient's satisfaction with the technology used. If patients are unhappy with the decision that is reached, it may be that expression of discontent with the videolink is a way of communicating their distress, without directly addressing the issue with their clinicians.

\section{AUTHOR'S CONTRIBUTIONS}

EM, DMB, FS \& CD participated in the conception of the study and its design and co-ordination. $\mathrm{CD}$ and EB performed data collection and EM carried out the data analysis, managed the literature searches and participated in the first drafting of the manuscript.

\section{ACKNOWLEDGEMENTS}

The questionnaires used were designed by Dr Saif Razvi, Consultant Neurologist, Institute of Neuroscience, Southern General Hospital, Glasgow. Input was also provided from Professor Victor Patterson, Chairman of Synapse Teleneurology Ltd. Slight modifications were made to ensure relevance to the bariatric population.

The Scottish Centre for Telehealth and NHS kindly allowed the use of their videoconferencing equipment and accommodation. The Scottish Centre for Telehealth also offered their expertise in facilitating remote clinical consultations

\section{REFERENCES}

[1] Buchwald H, Avidor Y, Braunwald E, et al. Bariatric surgery: a systematic review and meta-analysis. JAMA 2004; 292: 1724-37.

[2] Karlsson J, Taft C, Ryden A, Sjostrom L, Sullivan M. Ten-year trends in health-related quality of life after surgical and conventional treatment for severe obesity: the SOS intervention study. Int J Obes 2007; 31: 1248-61.

[3] Powers PS, Rosemurgy A, Boyd F, Perz A. Outcome of gastric restriction procedures: weight, psychiatric diagnosis and satisfaction. Obes Surg 1997; 7: 471-7.

[4] Larsen F. Psychosocial function before and after gastric banding surgery for morbid obesity: A prospective psychiatric study. Acta Psych Scand Suppl 1990; 359: 1-57.

[5] Kalarchian MA, Marcus MD, Levine MD, et al. Psychiatric disorders among bariatric surgery candidates: relationship to obesity and functional health status. Am J Psychiatry 2007; 164: 328-34.

[6] Mitchell JE, Lancaster KL, Burgard MA, et al. Long term follow up of patients' status after gastric bypass. Obes Surg 2001; 11: 13740.

[7] Marcus,MD, Kalarchian MA, Courcoulas AP. Psychiatric evaluation and follow up of bariatric surgery patients. Am J Psychiatry 2009; 166(3): 285-91.

[8] Kenkel J, Ed. Psychological considerations of the massive weight loss patient. Plast Reconst Surg 2006; 117(1): 17S-21S.

[9] Kinzel JF, Traweger C, Trefalt E, Biebl W. Psychosocial consequences of weight loss following gastric banding for morbid obesity. Obes Surg 2003; 13: 105-10.

[10] Ruskin PE, Silver-Aylaian M, Kling MA, et al. Treatment outcomes in depression: comparison of remote treatment through telepsychiatry to in-person treatment. Am J Psychiatry 2004; 161(8): 1471-7.

[11] Norman S. The use of telemedicine in psychiatry. J Psychiatr Ment Health Nurs 2006; 13: 771-7.

[12] O’Reilly R, Bishop J, Maddox K, Hutchinson L, Fisman M, Takhar J. Is telepsychiatry equivalent to face-to-face psychiatry. Results from a randomized controlled equivalence trial. Psychiatry Serv 2007; 58 (6): 836-43.

[13] Lexcen FJ, Hawk GL, Herrick S, Blank MB. Use of video conferencing for psychiatric and forensic evaluations. Psychiatry Serv 2006; 57(5): 713-5. 
[14] Simpson S, Deans G, Brebner E. The Delivery of a TelePsychology Service to Shetland. Clin Psychol Psychother 2001; 8: 130-5.

[15] Currell R, Urquhart, C, Wainwright P, Lewis R. Telemedicine versus face to face patient care: effects on professional practice and health care outcomes. Cochrane Database Syst Rev 2000, Issue 2: Art. No.: CD002098.

[16] Richardson LK, Frueh BC, Grubaugh AL, Egede L, Elhai JD. Current directions in videoconferencing tele-mental health research. Clin Psychol 2009; 1: 323-38.

[17] Harvey-Berino J. Changing health behaviour via telecommunications technology: using interactive television to treat obesity. Behav Ther 1998; 29: 505-19.

[18] Mattila E, Lappalainen R, Parkka J, Salminen J, Korhonen I. Use of a mobile phone diary for observing weight management and related behaviours. J Telemed Telecare 2010; 16: 260-4.
[19] Wu L, Forbes A, While A. Patients' experience of a telephone booster intervention to support weight management in Type 2 diabetes and its acceptability. J Telemed Telecare 2010; 16: 221-3.

[20] Shaikh U, Cole SL, Marcin JP, Nesbitt TS. Clinical management and patient outcomes among children and adolescents receiving telemedicine consultations for obesity. Telemed J E Health 2008; 14(5): 434-40.

[21] Haugen HA, Tran ZV, Wyatt HR, Barry MJ, Hill JO. Using telehealth to increase participation in weight maintenance programs. Obesity 2007; 15(12): 3067-77.

[22] Liou T, Chen C, Hsu C, Chou P, Chiu H. A pilot study of videoconferencing for an Internet-based weight loss programme for obese adults in Taiwan. J Telemed Telecare 2006; 12: 370-3.

[23] Goulis DG, Gigaglis GD, Boren SA, et al. Effectiveness of homecentered care through telemedicine applications for overweight and obese patients: a randomized controlled trial. Int J Obes 2004; 28 : 1391-8.

(C) Morrow et al.; Licensee Bentham Open.

This is an open access article licensed under the terms of the Creative Commons Attribution Non-Commercial License (http://creativecommons.org/licenses/by-nc/3.0/) which permits unrestricted, non-commercial use, distribution and reproduction in any medium, provided the work is properly cited 